\title{
Interhospital Transfer: Transfer Processes and Patient Outcomes
}

\author{
Stephanie K Mueller, MD, MPH ${ }^{1,2 \star}$; Julie Fiskio'; Jeffrey Schnipper, MD, MPH',2 \\ 'Division of General Internal Medicine, Brigham and Women's Hospital, Boston, Massachusetts; ${ }^{2}$ Harvard Medical School, Boston, Massachusetts.
}

Though often undertaken to provide patients with specialized care, interhospital transfer (IHT) is associated with worse outcomes for select patients. Certain aspects of the transfer process have been suggested as contributors to these outcomes. We performed a retrospective cohort study including patients $\geq 18$ years who underwent IHT to a tertiary care hospital between January 2005 and September 2013. We examined the association between "weekend" transfer, "nighttime" transfer, "time delay" between transfer acceptance and arrival, and admission team "busyness" on the day of transfer, and patient outcomes, including transfer to the intensive care unit (ICU) within 48 hours and 30-day mortality. We utilized multivariable logistic regression models, adjusting for patient characteristics. Secondary analyses examined detailed timing of transfer and evaluated 30-day mortality stratified by service of transfer. Among the 24,352 patients who underwent IHT, the nighttime transfer was associated with increased adjusted odds of ICU transfer (odds ratio [OR] 1.54; 95\% Cl 1.38, 1.72) and 30-day mortality (OR $1.16 ; 95 \% \mathrm{Cl} 1.01,1.35)$. Secondary analyses confirmed the association between nighttime transfer and ICU transfer throughout the week and demonstrated that Sunday (and trend towards Friday) night transfers had increased 30-day mortality, as compared with Monday daytime transfer. Stratified analyses demonstrated a significant association between transfer characteristics and adjusted odds of 30-day mortality among cardiothoracic and gastrointestinal surgical service transfers. Our findings suggest high acuity patients have worse outcomes during off-peak times of transfer and during times of high care team workload. Further study is needed to identify underlying reasons to explain these associations and devise potential solutions. Journal of Hospital Medicine 2019;14:486-491. Published April 8, 2019. @ 2019 Society of Hospital Medicine
T he transfer of patients between acute care hospitals (interhospital transfer $[\mathrm{IHT}]$ ) occurs regularly among patients with a variety of diagnoses, in theory, to gain access to unique specialty services and/or a higher level of care, among other reasons. ${ }^{1,2}$

However, the practice of IHT is variable and nonstandard$i_{i z e d, 3,4}^{3,4}$ and existing data largely suggests that transferred patients experience worse outcomes, including longer length of stay, higher hospitalization costs, longer ICU time, and greater mortality, even with rigorous adjustment for confounding by indication. 5,6 Though there are many possible reasons for these findings, existing literature suggests that there may be aspects of the transfer process itself which contribute to these outcomes. ${ }^{2,6,7}$

Understanding which aspects of the transfer process contribute to poor patient outcomes is a key first step toward the development of targeted quality improvement initiatives to improve this process of care. In this study, we aim to examine the association between select characteristics of the transfer process, including the timing of transfer and workload of the

*Corresponding Author: Stephanie Mueller, MD, MPH; E-mail: smueller1@ bwh.harvard.edu; Telephone: 617-278-0628

Additional Supporting Information may be found in the online version of this article.

Received: November 9, 2018; Revised: January 6, 2019; Accepted: February 15,2019

๑ 2019 Society of Hospital Medicine DOI 10.12788/jhm.3192 admitting physician team, and clinical outcomes among patients undergoing $\mathrm{IHT}$.

\section{METHODS}

\section{Data and Study Population}

We performed a retrospective analysis of patients zage 18 years who transferred to Brigham and Women's Hospital (BWH), a 777-bed tertiary care hospital, from another acute care hospital between January 2005, and September 2013. Dates of inclusion were purposefully chosen prior to BWH implementation of a new electronic health records system to avoid potential information bias. As at most academic medical centers, night coverage at BWH differs by service and includes a combination of long-call admitting teams and night float coverage. On weekends, many services are less well staffed, and some procedures may only be available if needed emergently. Some services have caps on the daily number of admissions or total patient census, but none have caps on the number of discharges per day. Patients were excluded from analysis if they left BWH against medical advice, were transferred from closely affiliated hospitals with shared personnel and electronic health records (Brigham and Women's Faulkner Hospital, Dana Farber Cancer Institute), transferred from inpatient psychiatric or inpatient hospice facilities, or transferred to obstetrics or nursery services. Data were obtained from administrative sources and the research patient data repository (RPDR), a centralized clinical data repository that gathers data from various hospital legacy systems and stores them in one data warehouse..$^{8}$ Our 
study was approved by the Partners Institutional Review Board (IRB) with a waiver of patient consent.

\section{Transfer Process Characteristics}

Predictors included select characteristics of the transfer process, including (1) Day of week of transfer, dichotomized into Friday through Sunday ("weekend"), versus Monday through Thursday ("weekday"); 9 Friday was included with "weekend" given the suggestion of increased volume of transfers in advance of the weekend; (2) Time of arrival of the transferred patient, categorized into "daytime" (7 AM-5 PM), "evening" (5 PM -10 PM), and "nighttime" (10 PM -7 AM), with daytime as the reference group; (3) Admitting team "busyness" on day of patient transfer, defined as the total number of additional patient admissions and patient discharges performed by the admitting team on the calendar day of patient arrival, as has been used in prior research, ${ }^{10}$ and categorized into quartiles with lowest quartile as the reference group. Service-specific quartiles were calculated and used for stratified analyses (described below); and (4) "Time delay" between patient acceptance for transfer and patient arrival at BWH, categorized into 0-12 hours, $12-24$ hours, 24-48 hours, and $>48$ hours, with $12-$ 24 hours as the reference group (anticipating that time delay of 0-12 hours would be reflective of "sicker" patients in need of expedited transfer).

\section{Outcomes}

Outcomes included transfer to the intensive care unit (ICU) within 48 hours of arrival and 30-day mortality from date of index admission. . $^{5}$

\section{Patient Characteristics}

Covariates for adjustment included: patient age, sex, race, Elixhauser comorbidity score, ${ }^{11}$ Diagnosis-Related Group (DRG)-weight, insurance status, year of admission, number of preadmission medications, and service of admission.

\section{Statistical Analyses}

We used descriptive statistics to display baseline characteristics and performed a series of univariable and multivariable logistic regression models to obtain the adjusted odds of each transfer process characteristic on each outcome, adjusting for all covariates (proc logistic, SAS Statistical Software, Cary, North Carolina). For analyses of ICU transfer within 48 hours of arrival, all patients initially admitted to the ICU at time of transfer were excluded.

In the secondary analyses, we used a combined day-of-week and time-of-day variable (ie, Monday day, Monday evening, Monday night, Tuesday day, and so on, with Monday day as the reference group) to obtain a more detailed evaluation of timing of transfer on patient outcomes. We also performed stratified analyses to evaluate each transfer process characteristic on adjusted odds of 30-day mortality stratified by service of admission (ie, at the time of transfer to BWH), adjusting for all covariates. For all analyses, two-sided $P$ values $<.05$ were considered significant.
TABLE 1. Baseline Characteristics of Transferred Patients

\begin{tabular}{|c|c|}
\hline Characteristic & $\begin{array}{l}\text { Transferred Patients } \\
\qquad(\mathrm{N}=24,352)\end{array}$ \\
\hline \multicolumn{2}{|l|}{ Patient Characteristics } \\
\hline Age in years, mean $(S D)^{a}$ & $62.2(16.3)$ \\
\hline Male sex, n (\%) & $13,647(56.0)$ \\
\hline $\begin{array}{l}\text { Race, } n(\%) \\
\text { White } \\
\text { Black } \\
\text { Hispanic } \\
\text { Other } \\
\end{array}$ & $\begin{array}{c}20,466(84.0) \\
759(3.1) \\
255(1.0) \\
2,872(11.8)\end{array}$ \\
\hline $\begin{array}{l}\text { Insurance, n (\%) } \\
\text { Medicare } \\
\text { Medicaid } \\
\text { Private } \\
\text { Other }\end{array}$ & $\begin{array}{c}13,231(54.3) \\
1,532(6.3) \\
8,958(36.8) \\
631(2.6)\end{array}$ \\
\hline $\begin{array}{l}\text { Admit year, } n(\%) \\
2005 \\
2006 \\
2007 \\
2008 \\
2009 \\
2010 \\
2011 \\
2012 \\
2013 \\
\end{array}$ & $\begin{array}{c}2,715(11.2) \\
2,768(11.4) \\
2,817(11.6) \\
2,777(11.4) \\
2,849(11.7) \\
2,805(11.5) \\
2,789(11.5) \\
2,730(11.2) \\
2,102(8.6) \\
\end{array}$ \\
\hline $\begin{array}{l}\text { Admission service, n (\%) } \\
\text { Cardiology } \\
\text { CT Surgery } \\
\text { Medicine }^{\mathrm{b}} \\
\text { Oncology/BMT } \\
\text { Neurology } \\
\text { ICUc } \\
\text { Orthopedic/Burn/Trauma } \\
\text { GI Surgery } \\
\text { Neurosurgery } \\
\text { Other }^{\mathrm{d}} \\
\end{array}$ & $\begin{array}{l}9,190(37.7) \\
3,156(13.0) \\
2,466(10.1) \\
2,183(9.0) \\
1,456(6.0) \\
1,442(5.9) \\
1,235(5.1) \\
1,066(4.4) \\
768(3.2) \\
912(3.7) \\
\end{array}$ \\
\hline Elixhauser comorbidity score, mean (SD) a & $7.2(7.8)$ \\
\hline DRG-weight, mean (SD) & $2.8(2.9)$ \\
\hline $\begin{array}{l}\text { Number of preadmission medications quartile } \\
0-1 \\
2-6 \\
7-10 \\
\geq 11 \\
\text { Missing patient data }\end{array}$ & $\begin{array}{l}5,225(21.5) \\
5,777(23.7) \\
4,549(18.7) \\
4,677(19.2) \\
4,124(16.9)\end{array}$ \\
\hline Transfer Process Characteristics & \\
\hline Weekday transfer (Monday-Thursday), n (\%) & $14,612(60.0)$ \\
\hline $\begin{array}{l}\text { Time of day of transfer, } \mathrm{n}(\%) \\
\text { Daytime (7 AM - } 5 \text { PM) } \\
\text { Evening (5 PM - } 10 \text { PM) } \\
\text { Nighttime (10 PM - } 7 \text { AM) }\end{array}$ & $\begin{array}{c}7,917(32.5) \\
12,597(51.7) \\
3,838(15.8)\end{array}$ \\
\hline $\begin{array}{l}\text { Admission team busyness }{ }^{e} \text { on day of patient } t \\
0-4 \\
5-7 \\
8-10 \\
\geq 10\end{array}$ & $\begin{array}{l}5,393(22.2) \\
7,382(30.3) \\
6,012(24.7) \\
5,565(22.8)\end{array}$ \\
\hline $\begin{array}{l}\text { Time delay between transfer acceptance and } \\
0-12 \text { hours } \\
>12-24 \text { hours } \\
>24-48 \text { hours } \\
>48 \text { hours }\end{array}$ & $\begin{array}{c}17,896(74.3) \\
1,766(7.3) \\
3,080(12.8) \\
1,336(5.5)\end{array}$ \\
\hline
\end{tabular}

${ }^{\mathrm{a}}$ Categorized into quartiles for multivariable regression analyses

bMedicine service includes: General medicine, gastroenterology, renal, endocrine, hypertension, infectious disease, and rheumatology services.

${ } \mathrm{CU}$ patients were excluded in all regression analyses examining odds of ICU transfer within 48 hours of admission

'Other service includes: otolaryngology, urology, plastic surgery, gynecology, dental, and other services

eBusyness defined as total number of other patient admissions and discharges by admission team on day of patient transfer

Abbreviations: BMT, bone marrow transplant; $\mathrm{CT}$, cardiothoracic; DRG, diagnosis-related group; $\mathrm{Gl}$, gastrointestinal; ICU, intensive care unit; SD, standard deviation. 
TABLE 2. Association of Transfer Process Characteristics and Adjusted Odds of ICU Transfer and 30-Day Mortality

\begin{tabular}{|c|c|c|c|}
\hline Transfer Process Characteristic & ICU Transfer within 48 hours, $\mathrm{n}(\%)^{\mathrm{a}}$ & Unadjusted OR $(95 \% \mathrm{Cl})^{\mathrm{b}}$ & Adjusted OR $(95 \% \mathrm{Cl})$ \\
\hline \multicolumn{4}{|l|}{ Day of Week of Arrival } \\
\hline Weekend, $n=9,088$ & $2,020(22 \%)$ & $0.83(0.77,0.88)$ & $0.93(0.87,1.01)$ \\
\hline Weekday, $n=13,774$ & $3,598(26 \%)$ & Ref & Ref \\
\hline \multicolumn{4}{|l|}{ Time of Day of Arrival } \\
\hline Nighttime, $n=3,449$ & $1,233(36 \%)$ & $1.57(1.43,1.72)$ & $1.54(1.38,1.72)$ \\
\hline Daytime, $\mathrm{n}=7,521$ & $1,813(24 \%)$ & Ref & Ref \\
\hline $0-4, n=7,399$ & $2,448(33 \%)$ & Ref & Ref \\
\hline \multicolumn{4}{|l|}{ Time Delay between Acceptance/Arrival } \\
\hline$>48$ hours & $172(13.2 \%)$ & $1.00(0.81,1.24)$ & $0.86(0.67,1.08)$ \\
\hline $12-24$ hours & $239(14 \%)$ & Ref & Ref \\
\hline Transfer Process Characteristic & 30-Day Mortality, n (\%) & Unadjusted OR $(95 \% \mathrm{Cl})$ & Adjusted OR $(95 \% \mathrm{Cl})^{\mathrm{a}}$ \\
\hline \multicolumn{4}{|l|}{ Time of Day of Arrival } \\
\hline Nighttime, $n=3,828$ & $449(12 \%)$ & $1.67(1.46,1.90)$ & $1.16(1.01,1.35)$ \\
\hline Daytime, $n=7,894$ & $565(7.2 \%)$ & Ref & Ref \\
\hline \multicolumn{4}{|l|}{ Admitting team busyness } \\
\hline$\geq 10, n=5,414$ & $389(7.2 \%)$ & $0.77(0.67,0.87)$ & $0.89(0.77,1.02)$ \\
\hline $0-4, n=7,966$ & $749(9.4 \%)$ & Ref & Ref \\
\hline \multicolumn{4}{|l|}{ Time Delay between Acceptance/Arrival } \\
\hline$>48$ hours & $108(8.1 \%)$ & $0.97(0.74,1.26)$ & $0.88(0.66,1.17)$ \\
\hline $12-24$ hours & $135(7.6 \%)$ & Ref & Ref \\
\hline
\end{tabular}

${ }^{\mathrm{a} E x c l u d e d}$ ICU service patients

${ }^{b}$ Adjusted for all patient characteristics (Table), and all other transfer process characteristics

Abbreviations: Cl, confidence interval; ICU, intensive care unit; OR, odds ratio; Ref, referent.

Definitions: Weekday, Monday through Thursday; Weekend, Friday through Sunday; Nighttime, 10 PM - 7 AM, Daytime = 7 AM - 5 PM; Admitting team busyness, Number of additional patient admissions + discharges performed by admitting team on day of patient arrival.

\section{RESULTS}

Overall, 24,352 patients met our inclusion criteria and underwent IHT, of whom 2,174 (8.9\%) died within 30 days. Of the 22,910 transferred patients originally admitted to a non-ICU service, 5,464 (23.8\%) underwent ICU transfer within 48 hours of arrival. Cohort characteristics are shown in Table 1.

Multivariable regression analyses demonstrated no significant association between weekend (versus weekday) transfer or increased time delay between patient acceptance and arrival (>48 hours) and adjusted odds of ICU transfer within 48 hours or 30-day mortality. However, they did demonstrate that nighttime (versus daytime) transfer was associated with greater adjusted odds of both ICU transfer and 30-day mortality. Increased admitting team busyness was associated with lower adjusted odds of ICU transfer but was not significantly associated with adjusted odds of 30-day mortality (Table 2). As expected, decreased time delay between patient acceptance and arrival (0-12 hours) was associated with increased adjusted odds of both ICU transfer (adjusted OR 2.68; 95\% Cl 2.29, 3.15 ) and 30-day mortality (adjusted OR 1.25; $95 \% \mathrm{Cl} 1.03,1.53$ ) compared with 12-24 hours (results not shown). Time delay $>48$ hours was not associated with either outcome.

Regression analyses with the combined day/time variable demonstrated that compared with Monday daytime transfer, Sunday night transfer was significantly associated with increased adjusted odds of 30-day mortality, and Friday night transfer was associated with a trend toward increased 30-day mortality (adjusted OR [aOR] 1.88; 95\% Cl 1.25, 2.82, and aOR $1.43 ; 95 \% \mathrm{Cl} 0.99,2.06$, respectively). We also found that all nighttime transfers (ie, Monday through Sunday night) were associated with increased adjusted odds of ICU transfer within 48 hours (as compared with Monday daytime transfer). Other days/time analyses were not significant.

Univariable and multivariable analyses stratified by service were performed (Appendix). Multivariable stratified analyses demonstrated that weekend transfer, nighttime transfer, and increased admitting team busyness were associated with increased adjusted odds of 30-day mortality among cardiothoracic $(C T)$ and gastrointestinal (GI) surgical service patients. Increased admitting team busyness was also associated with increased mortality among ICU service patients but was associated with decreased mortality among cardiology service patients. An increased time delay between patient acceptance and arrival was associated with decreased mortality among CT and GI surgical service patients (Figure; Appendix). Other adjusted stratified outcomes were not significant. 

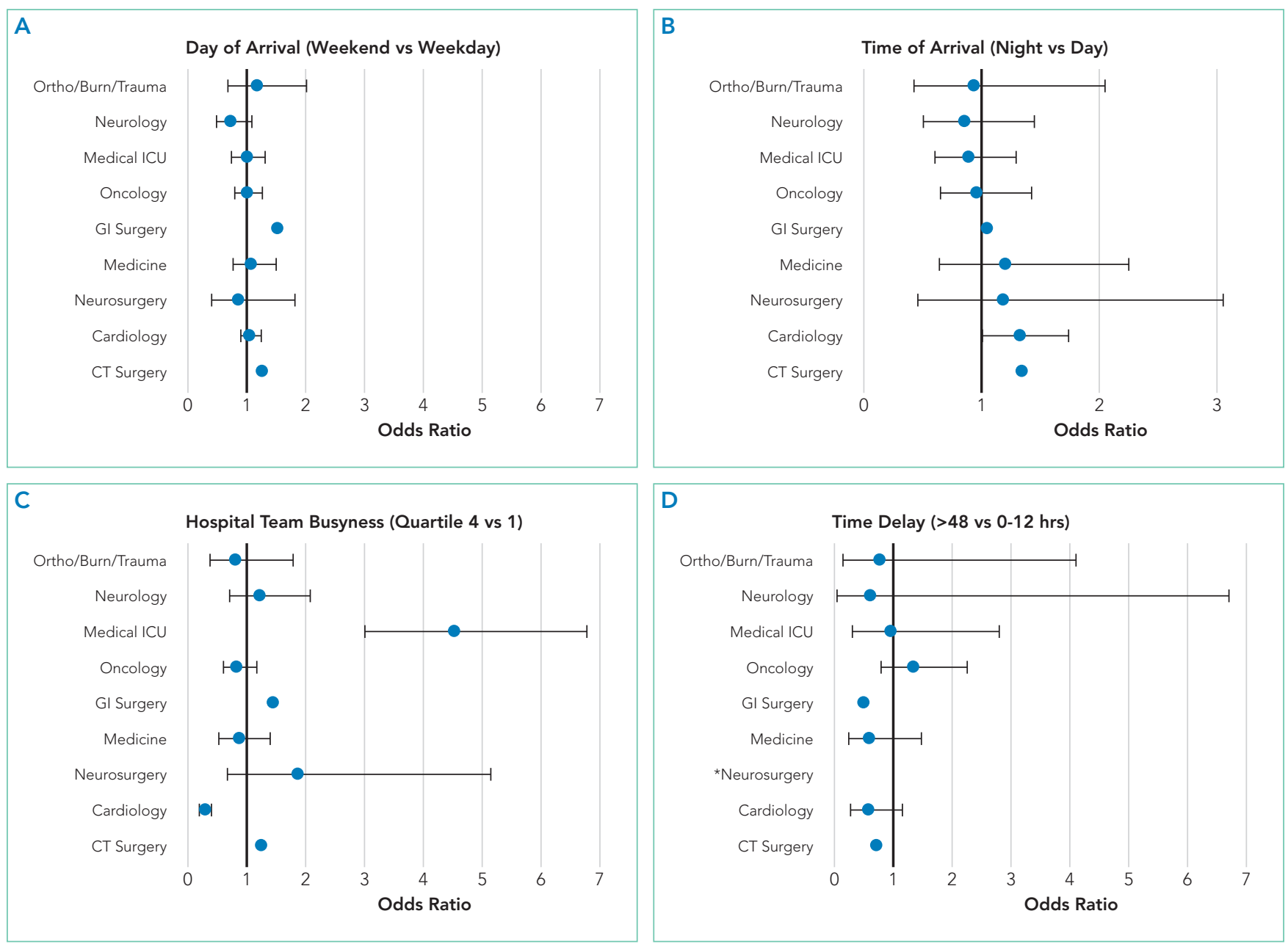

FIG. Association of Transfer Process Characteristic with Adjusted Odds of 30-day Mortality Stratified by Service.

*Too few outcomes to perform stratified analyses for association of increased time delay and adjusted odds of 30-day mortality among neurosurgical service transfers Service-specific quartiles were calculated and used for Hospital Team busyness analysis (Appendix)

Abbreviations: CT, Cardiothoracic; GI, Gastrointestinal; ICU, Intensive Care Unit; Ortho, Orthopedics

\section{DISCUSSION}

In this study of 24,352 patients undergoing $\mathrm{IHT}$, we found no significant association between weekend transfer or increased time delay between transfer acceptance and arrival and patient outcomes in the cohort as a whole; but we found that nighttime transfer is associated with increased adjusted odds of both ICU transfer within 48 hours and 30-day mortality. Our analyses combining day-of-week and time-of-day demonstrate that Sunday night transfer is particularly associated with increased adjusted odds of 30-day mortality (as compared with Monday daytime transfer), and show a trend toward increased mortality with Friday night transfers. These detailed analyses otherwise reinforce that nighttime transfer across all nights of the week is associated with increased adjusted odds of ICU transfer within 48 hours. We also found that increased admitting team busyness on the day of patient transfer is associated with decreased odds of ICU transfer, though this may solely be reflective of higher turnover services (ie, cardiology) caring for lower acuity patients, as suggested by secondary analyses stratified by service. In addition, secondary analyses demon- strated differential associations between weekend transfers, nighttime transfers, and increased team busyness on the odds of 30-day mortality based on service of transfer. These analyses showed that patients transferred to higher acuity services requiring procedural care, including CT surgery, Gl surgery, and Medical ICU, do worse under all three circumstances as compared with patients transferred to other services. Secondary analyses also demonstrated that increased time delay between patient acceptance and arrival is inversely associated with 30day mortality among CT and GI surgery service patients, likely reflecting lower acuity patients (ie, less sick patients are less rapidly transferred).

There are several possible explanations for these findings. Patients transferred to surgical services at night may reflect a more urgent need for surgery and include a sicker cohort of patients, possibly explaining these findings. Alternatively, or in addition, both weekend and nighttime hospital admission expose patients to similar potential risks, ie, limited resources available during off-peak hours. Our findings could, therefore, reflect the possibility that patients transferred to higher acui- 
ty services in need of procedural care are most vulnerable to off-peak timing of transfer. Similar data looking at patients admitted through the emergency room (ER) find the strongest effect of off-peak admissions on patients in need of procedures, including $\mathrm{Gl}$ hemorrhage, ${ }^{12}$ atrial fibrillation ${ }^{13}$ and acute myocardial infarction (AMI), ${ }^{14}$ arguably because of the limited availability of necessary interventions. Patients undergoing IHT are a sicker cohort of patients than those admitted through the $E R$, and, therefore, may be even more vulnerable to these issues. ${ }^{3,5}$ This is supported by our findings that Sunday night transfers (and trend toward Friday night transfers) are associated with greater mortality compared with Monday daytime transfers, when at-the-ready resources and/or specialty personnel may be less available (Sunday night), and delays until receipt of necessary procedures may be longer (Friday night). Though we did not observe similar results among cardiology service transfers, as may be expected based on existing literature, ${ }^{13,14}$ this subset of patients includes more heterogeneous diagnoses, (ie, not solely those that require acute intervention) and exhibited a low level of acuity (low Elixhauser score and DRG-weight, data not shown).

We also found that increased admitting team busyness on the day of patient transfer is associated with increased odds of 30-day mortality among CT surgery, GI surgery, and ICU service transfers. As above, there are several possible explanations for this finding. It is possible that among these services, only the sickest/neediest patients are accepted for transfer when teams are busiest, explaining our findings. Though this explanation is possible, the measure of team "busyness" includes patient discharge, thereby increasing, not decreasing, availability for incoming patients, making this explanation less likely. Alternatively, it is possible that this finding is reflective of reverse causation, ie, that teams have less ability to discharge/ admit new patients when caring for particularly sick/unstable patient transfers, though this assumes that transferred patients arrive earlier in the day, (eg, in time to influence discharge decisions), which infrequently occurs (Table 1). Lastly, it is possible that this subset of patients will be more vulnerable to the workload of the team that is caring for them at the time of their arrival. With high patient turnover (admissions/discharges), the time allocated to each patient's care may be diminished (ie, "work compression," trying to do the same amount of work in less time), and may result in decreased time to care for the transferred patient. This has been shown to influence patient outcomes at the time of patient discharge. ${ }^{10}$

In trying to understand why we observed an inverse relationship between admitting team busyness and odds of ICU transfer within 48 hours, we believe this finding is largely driven by cardiology service transfers, which comprise the highest volume of transferred patients in our cohort (Table 1), and are low acuity patients. Within this population of patients, admitting team busyness is likely a surrogate variable for high turnover/low acuity. This idea is supported by our findings that admitting team busyness is associated with decreased adjusted odds of 30-day mortality in this group (and only in this group).

Similarly, our observed inverse relationship between in- creased time delay and 30-day mortality among CT and GI surgical service patients is also likely reflective of lower acuity patients. We anticipated that decreased time delay (0-12 hours) would be reflective of greater patient acuity (supported by our findings that decreased time delay is associated with increased odds of ICU transfer and 30-day mortality). However, our findings also suggest that increased time delay ( $>48$ hours) is similarly representative of lower patient acuity and therefore an imperfect measure of discontinuity and/or harmful delays in care during $\mathrm{IHT}$ (see limitations below).

Our study is subject to several limitations. This is a single site study; given known variation in transfer practices between hospitals, ${ }^{3}$ it is possible that our findings are not generalizable. However, given similar existing data on patients admitted through the ER, it is likely our findings may be reflective of IHT to similar tertiary referral hospitals. Second, although we adjusted for patient characteristics, there remains the possibility of unmeasured confounding and other bias that account for our results, as discussed. Third, although the definition of "busyness" used in this study was chosen based on prior data demonstrating an effect on patient outcomes, ${ }^{10}$ we did not include other measures of busyness that may influence outcomes of transferred patients such as overall team census or hospital busyness. However, the workload associated with a high volume of patient admissions and discharges is arguably a greater reflection of "work compression" for the admitting team compared with overall team census, which may reflect a more static workload with less impact on the care of a newly transferred patient. Also, although hospital census may influence the ability to transfer (ie, lower volume of transferred patients during times of high hospital census), this likely has less of an impact on the direct care of transferred patients than the admitting team's workload. It is more likely that it would serve as a confounder (eg, sicker patients are accepted for transfer despite high hospital census, while lower risk patients are not).

Nevertheless, future studies should further evaluate the association with other measures of busyness/workload and outcomes of transferred patients. Lastly, though we anticipated time delay between transfer acceptance and arrival would be correlated with patient acuity, we hypothesized that longer delay might affect patient continuity and communication and impact patient outcomes. However, our results demonstrate that our measurement of this variable was unsuccessful in unraveling patient acuity from our intended evaluation of these vulnerable aspects of IHT. It is likely that a more detailed evaluation is required to explore potential challenges more fully that may occur with greater time delays (eg, suboptimal communication regarding changes in clinical status during this time period, delays in treatment). Similarly, though our study evaluates the association between nighttime and weekend transfer (and the interaction between these) with patient outcomes, we did not evaluate other intermediate outcomes that may be more affected by the timing of transfer, such as diagnostic errors or delays in procedural care, which warrant further investigation. We do not directly examine the underly- 
ing reasons that explain our observed associations, and thus more research is needed to identify these as well as design and evaluate solutions.

Collectively, our findings suggest that high acuity patients in need of procedural care experience worse outcomes during off-peak times of transfer, and during times of high care-team workload. Though further research is needed to identify underlying reasons to explain our findings, both the timing of patient transfer (when modifiable) and workload of the team caring for the patient on arrival may serve as potential targets for interventions to improve the quality and safety of IHT for patients at greatest risk.

Disclosures: Dr. Mueller and Dr. Schnipper have nothing to disclose. Ms. Fiskio has nothing to disclose. Dr. Schnipper is the recipient of grant funding from Mallinckrodt Pharmaceuticals to conduct an investigator-initiated study of predictors and impact of opioid-related adverse drug events.

\section{References}

1. Iwashyna TJ. The incomplete infrastructure for interhospital patient transfer. Crit Care Med. 2012;40(8):2470-2478. https://doi.org/10.1097/ CCM.0b013e318254516f.

2. Mueller SK, Shannon E, Dalal A, Schnipper JL, Dykes P. Patient and physician experience with interhospital transfer: a qualitative study. J Patient Saf. 2018. https://doi.org/10.1097/PTS.0000000000000501

3. Mueller SK, Zheng J, Orav EJ, Schnipper JL. Rates, predictors and variability of interhospital transfers: a national evaluation. J Hosp Med. 2017;12(6):435-442. https://doi.org/10.12788/jhm.2747.

4. Bosk EA, Veinot T, Iwashyna TJ. Which patients and where: a qualitative study of patient transfers from community hospitals. Med Care. 2011;49(6):592-598. https://doi.org/10.1097/MLR.0b013e31820fb71b.

5. Sokol-Hessner L, White AA, Davis KF, Herzig SJ, Hohmann SF. Interhospital transfer patients discharged by academic hospitalists and general internists: characteristics and outcomes. J Hosp Med. 2016;11(4):245-50. https://doi. org/10.1002/jhm.2515.

6. Mueller S, Zheng J, Orav EJP, Schnipper JL. Inter-hospital transfer and patient outcomes: a retrospective cohort study. BMJ Qual Saf. 2018. https:// doi.org/10.1136/bmjqs-2018-008087.

7. Mueller SK, Schnipper JL. Physician perspectives on interhospital transfers. J Patient Saf. 2016. https://doi.org/10.1097/PTS.0000000000000312.

8. Research Patient Data Registry (RPDR). http://rc.partners.org/rpdr. Accessed April 20, 2018.

9. Bell CM, Redelmeier DA. Mortality among patients admitted to hospitals on weekends as compared with weekdays. N Engl J Med. 2001;345(9):663-668. https://doi.org/10.1056/NEJMsa003376

10. Mueller SK, Donze J, Schnipper JL. Intern workload and discontinuity of care on 30-day readmission. Am J Med. 2013;126(1):81-88. https://doi. org/10.1016/j.amjmed.2012.09.003.

11. Elixhauser A, Steiner C, Harris DR, Coffey RM. Comorbidity measures for use with administrative data. Med Care. 1998;36(1):8-27.

12. Ananthakrishnan AN, McGinley EL, Saeian K. Outcomes of weekend admissions for upper gastrointestinal hemorrhage: a nationwide analysis. Clin Gastroenterol Hepatol. 2009;7(3):296-302e1. https://doi.org/10.1016/j.cgh.2008.08.013.

13. Deshmukh A, Pant S, Kumar G, Bursac Z, Paydak H, Mehta JL. Comparison of outcomes of weekend versus weekday admissions for atrial fibrillation. Am J Cardiol. 2012;110(2):208-211. https://doi.org/10.1016/j.amjcard.2012.03.011.

14. Clarke MS, Wills RA, Bowman RV, et al. Exploratory study of the 'weekend effect' for acute medical admissions to public hospitals in Queensland, Australia. Intern Med J. 2010;40(11):777-783. https://doi.org/10.1111/j.14455994.2009.02067.x. 